Website: http://revistas.lamolina.edu.pe/index.php/acu/index

(C) Universidad Nacional Agraria La Molina, Lima - Perú

\title{
Injerto de granadilla (Passiflora ligularis Juss.) sobre maracuyá (Passiflora edulis $f$. flavicarpa) utilizando dos secciones de brotes de plantas adultas, dos tipos de injerto y dos cámaras húmedas individuales
}

\author{
Grafting of two shoot segments from granadilla adult plants (passiflora ligularis Juss.) onto maracuyá \\ (Passiflora edulis $f$. flavicarpa) using two grafting types and two individual humid chambers
}

\author{
Enrique Cuya $\mathrm{Curo}^{1} \&$ Jorge Escobedo Alvarez ${ }^{2 *}$ \\ * Autor de correspondencia
}

\section{Resumen}

Con el objetivo de evaluar la propagación por injerto de granadilla (Passiflora ligularis Juss.) sobre maracuyá (Passiflora edulis f. flavicarpa) en vivero, se estudiaron dos formas de injerto: hendidura e inglés simple, dos tipos de estaca o pluma: apical y sub-apical y dos tipos de cámara húmeda individual: envase plástico transparente de bebidas y bolsa de polietileno transparente. Los portainjertos fueron plántulas de semilla, de 40-47 días de edad y las plumas se confeccionaron de brotes herbáceos de plantas de dos años (las apicales de 12-15 cm de longitud, contenían el ápice del brote y 3-4 nudos con hojas completamente expandidas y las sub-apicales de 10-14 cm, solo poseían los 3-4 nudos con hojas). Después de cada injerto se cubrió la planta con la respectiva cámara húmeda individual. El prendimiento de los injertos, 32 días después, fue más elevado con el de hendidura (44,99\%) que con el inglés simple $(31,65 \%)$; por su parte, con plumas sub-apicales el prendimiento fue nulo, mientras que con las apicales se logró 76,64\%; finalmente, la cámara húmeda individual conformada por envase de bebidas con 41,65 \% fue mejor que la bolsa de polietileno que registró 34,99 \%. El tamaño del brote del injerto, 67 días después de la injertación mostró el mismo orden de eficiencia anotado para el prendimiento: injerto de hendidura mejor que inglés simple $(21,65$ y 13,28 cm), la pluma apical, como único tipo que tuvo prendimiento, registro $34,93 \mathrm{~cm}$, y el envase de bebida como cámara húmeda alcanzó $20,27 \mathrm{~cm}$, en tanto bolsa de polietileno logró $14,65 \mathrm{~cm}$.

Palabras clave: granadilla; maracuyá; injerto; pluma, cámara húmeda; prendimiento del injerto.

\begin{abstract}
Whith objetive to assess the propagating granadilla (Passiflora ligularis Juss.) by grafting on passion fruit (Passiflora edulis f. flavicarpa) in nursery, were evaluated: two graft types cleft and splice, two different shoots segments for grafting: apical and sub-apical, and two types of individual humid chamber: transparent plastic containers and transparent plastic bags. Rootstocks were seedlings 40-47 days old, herbaceous stems segments used for grafting came from two years old plants (apical portions had 12-15 cm in length containing the shoot apex and 3-4 nodes with fully expanded leaves, sub-apical portions had 10-14 cm, only with 3-4 nodes and leaves). After each graft was made, the plant was covered immediately with its respective humid chamber. Percentage of successful grafts, 32 days later, was higher with the cleft graft method $(44,99 \%)$ than with the splice graft $(31,65 \%)$; on the other hand, all the sub-apical segments grafts did not survive, while the apical ones achieved 76,64\% success; finally, as individual humid chamber plastic containers with $41,65 \%$ survival rate resulted in higher success than plastic bags (34,99\%). Evaluating the size of the new shoot 67 days after grafting, the same previous order of efficiency was observed: cleft better than splice graft $(21,65$ and $13,28 \mathrm{~cm}$ long), the apical portions of shoots, the only type of shoot segment that survived was $34,93 \mathrm{~cm}$ long, and the shoot length in plastic containers as humid chamber reached $20,27 \mathrm{~cm}$, while plastic bag only achieved $14,65 \mathrm{~cm}$.
\end{abstract}

Keywords: granadilla; passion fruit; graft; shoot segments; humid chamber; grafting success.

\section{Introducción}

En el Perú, las especies del género Passiflora están ampliamente distribuidas, desde la costa a nivel del mar hasta las zonas montañosas de los andes y también en la selva tropical de la Amazonía. En efecto, Esquerre-Ibáñez et al. (2014) consideran que aquí existen alrededor de 100 especies endémicas de este género. Como en el resto de países americanos donde se la cultiva, comercialmente, la granadilla (Passiflora ligularis Juss.) es la segunda especie en importancia, después del maracuyá amarillo (Passiflora edulis f. flavicarpa). 
El método más utilizado para obtener plantas definitivas de granadilla, al igual que para otras pasifloráceas es la propagación por semilla botánica, que con algunos tratamientos especiales es relativamente sencillo de realizar y da lugar a plantas vigorosas, con buena formación de sistema radicular (Cárdenas, 2011), sin embargo debido a la polinización cruzada de la especie, las plantas muestran una gran variabilidad genética entre ellas, que confiere desuniformidad a la plantación (Cerdas y Castro, 2003), además de muchos otros inconvenientes que se generan por la susceptibilidad de la granadilla a factores adversos del suelo como los nematodes y las pudriciones radiculares a los que la granadilla es susceptible. Precisamente para hacer frente a estos y otros problemas, la propagación por injerto aparece como la alternativa más eficiente pues además de permitir la propagación de genotipos superiores y posibilitar el uso de portainjertos tolerantes o resistentes a condiciones limitantes del suelo, se puede igualmente controlar el tamaño de las plantas reducir la juvenilidad y mejorara algunos atributos de calidad de la fruta (Ruggiero y Oliveira, 1998). Esto plantea la necesidad de encontrar metodologías que permitan la propagación comercial de la granadilla por injerto. En el Perú se utiliza a pequeña escala desde hace unos 12- 15 años el injerto de aproximación empleando pequeñas plantas de maracuyá originada de semilla como portainjertos e igualmente plantitas de origen sexual de granadilla como injerto, ambos creciendo en el mismo contenedor. Con el mismo tipo de plántulas pero empleando la parte apical (epicotiledonar) como pluma o púa de la plantita a propagar que será insertada en la zona hipocotiledonar de la otra plántula que es el portainjerto, constituye lo que se denomina el método del injerto hipocotiledonar, que se reporta como una buena alternativa para propagar granadilla sobre maracuyá (Maicelo et al., 2017) y también maracuyá amarillo sobre otras especies del género Passiflora (Zucareli et al., 2014). Los tipos de injerto que con mayor frecuencia se indican como los más eficientes son el de hendidura y el inglés simple, así como también se resaltan efectos positivos de las cámaras húmedas en el prendimiento de los injertos (Santos et al., 2011; Santos et al., 2014: Silva et al., 2015; Nogueira et al., 2011). Otra variante interesante de esta técnica o procedimiento que se ha experimentado en maracuyá amarillo con diversos porta injertos, consiste en usar como plumas portadoras de yemas, pequeñas secciones apicales de plantas adultas que se injertan en la parte apical seccionada de los hipocótilos, a través del injerto de hendidura, constituyendo lo que se conoce como "miniinjertos" (Alexandre et al., 2013; Oliari et al., 2016).

La presente investigación se planificó con la finalidad de evaluar a nivel de vivero, el injerto de granadilla empleando como plumas, conteniendo las yemas, porciones de entre 10 y $15 \mathrm{~cm}$ de longitud, de ramas de plantas en producción y como portainjertos plántulas de semilla de maracuyá de 40-47 días de edad y 18-20 cm de altura, en función de tres variables: porción del brote empleado como pluma: apical y sub-apical, y tipo de cámara húmeda individual: bolsa de polietileno transparente y envase plástico de bebidas.

\section{Materiales y métodos}

\section{Lugar experimental y tratamientos}

El experimento se llevó a cabo en el vivero del Programa de Investigación y Proyección Social de Frutales de la Universidad Nacional Agraria La Molina, entre el 1 de febrero y el 07 de junio del 2011. Los tratamientos considerados fueron consecuencia de la combinación de tres factores y dos niveles de cada factor, de acuerdo al siguiente detalle: Factor 1 (Tipo de injerto: hendidura e inglés simple), Factor 2 (Pluma empleada para el injerto; apical y sub-apical) y Factor 3 (Cámara húmeda individual: bolsa plástica y envase de bebidas). Como consecuencia, los tratamientos resultantes fueron 8. T1 (injerto de hendidura, pluma apical, bolsa de polietileno), T2 (injerto inglés simple), T3 (injerto de hendidura, pluma sub-apical), T4 (injerto inglés simple), T5 (injerto de hendidura, pluma apical, envase de bebida), T6 (injerto inglés simple), T7 (injerto de hendidura, pluma sub-apical) y T8 (injerto inglés simple)

\section{Diseño experimental}

Los tratamientos fueron distribuidos de acuerdo a un diseño completamente al azar (DCA) con arreglo factorial. Se emplearon un total de 30 plantas por tratamiento, distribuidas en 5 repeticiones de seis plantas cada una.

\section{Materiales de propagación, procedimientos}

Los portainjertos fueron plantas de semilla de una selección local de maracuyá amarillo, creciendo en contenedores plásticos de 5 litros de capacidad con un sustrato compuesto por arena $(50 \%)$, turba de Distichia sp. $(20 \%)$, tierra de chacra $(20 \%)$ y compost $(10 \%)$. Los ápices herbáceos de los que se confeccionaron las plumas o púas para los injertos, se colectaron de plantas de dos años de un biotipo igualmente local de granadilla, conducidas como plantas francas.

Todos los injertos fueron hechos a una altura de 10$13 \mathrm{~cm}, 60$ días después de la siembra de las semillas de los portainjertos, cuando las plantitas tenían entre 40 y 47 días de edad y aproximadamente $20 \mathrm{~cm}$ de altura y $0,5 \mathrm{~cm}$ de diámetro. Las plumas o estacas que son los segmentos de tallo que contienen las yemas, fueron de dos clases: apicales, de entre 12 y $15 \mathrm{~cm}$ de longitud que contenían el ápice del brote y 3-4 nudos con hojas desarrolladas y las sub apicales de 10 a $14 \mathrm{~cm}$ con solo las 3-4 hojas.

Como cámaras húmedas individuales se emplearon envases plásticos transparentes de bebidas de $500 \mathrm{cc}$ de capacidad, cortados en su parte basal, y bolsas plásticas transparentes que fueron colocadas sobre las plantas inmediatamente después de realizado cada injerto: la parte basal de los envases de bebidas se introdujo $0,5-0,8 \mathrm{~cm}$ en el sustrato del contenedor y la parte basal abierta, de las bolsas plásticas, sin atar, quedaron hasta $3 \mathrm{~cm}$ sobre la superficie del mismo. Las cámaras húmedas fueron retiradas una vez que se verificaba el prendimiento del injerto. 


\section{Evaluaciones}

La primera evaluación se realizó 32 días después de la injertación y se determinó el prendimiento de los injertos, considerándose como evidencia de éxito la presencia de brote o brotes nuevos sobre la pluma, luego se procedió a dejar un solo brote. Posteriormente, 35 días más tarde (67 días después de los injertos) se midió la longitud del nuevo brote apical generado. Con los datos colectados se realizó el análisis de varianza (ANVA) de un diseño tri factorial con el programa estadístico $\mathrm{R}$ y se usó la prueba de comparación de medias Tukey $(\mathrm{p}<0,05)$ de las fuentes de variación.

\section{Resultados y discusión}

\section{Prendimiento de los injertos}

El efecto combinado o interacción de los tres factores en el éxito de los injertos se muestra en la Figura 1, donde se puede observar que con los tratamientos T3, T4, T7 y T8, en los que se usó segmentos sub-apicales como plumas, el prendimiento fue nulo.

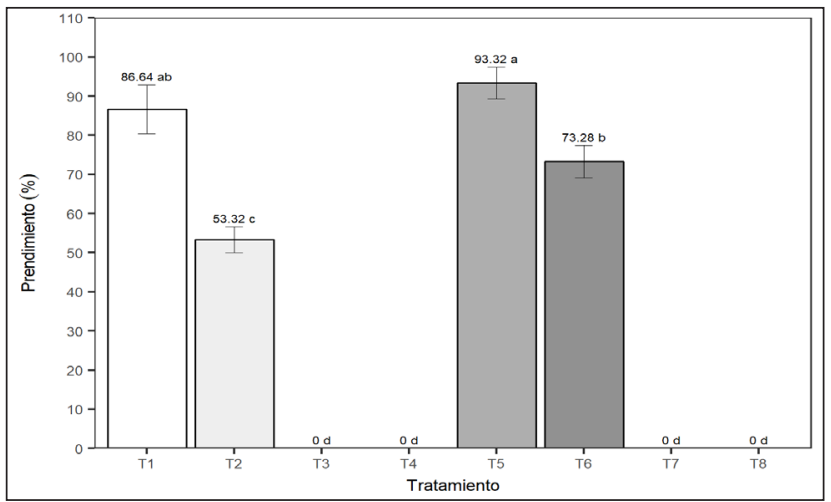

Figura 1. Porcentaje de prendimiento de los injertos de granadilla sobre maracuyá 32 días después de efectuados. Interacción de forma de injerto, tipo de pluma y tipo de cámara húmeda

T1: injerto hendidura + pluma apical + bolsa de polietileno, $\mathrm{T} 2$ : injerto inglés simple + pluma apical + bolsa de polietileno, T3: injerto hendidura + pluma sub-apical + bolsa de polietileno, T4: injerto inglés simple + pluma sub-apical + bolsa de polietileno, T5: injerto hendidura + pluma apical + envase de bebida, T6: injerto inglés simple + pluma apical + envase de bebida, T7: injerto Hendidura + pluma sub-apical + envase de bebida, T8: injerto inglés simple + pluma sub-apical + envase de bebida.

Los otros cuatro tratamientos mostraron buenos porcentajes de éxito de los injertos, destacando T5, T1 y T6 (con 93,32, 86,64 y 73,28\% respectivamente) sobre T2 que alcanzó 53,32\%.

En la Figura 2 los promedios o efectos simples de cada factor confirman que efectivamente con las plumas subapicales no se alcanzó ningún injerto prendido, mientras que con los segmentos apicales se registró $76,64 \%$ de prendimiento. Esto no concuerda con los resultados obtenidos por Silva et al. (2005) que con plumas de la parte apical y de la parte intermedia de los brotes obtuvo a los 50 días de realizados los injertos de maracuyá amarillo sobre
Passiflora alata, el mismo porcentaje de sobrevivencia de losinjertos (entre 78,4 y 86,4\% para pluma apical y entre 86,4 y $96,8 \%$ para pluma sub-apical). Si la viabilidad y capacidad de brotamiento de las yemas axilares de la granadilla son similares al maracuyá, el fracaso de las plumas sub-apicales en el presente experimento podría ser consecuencia de la acumulación de etileno que pudo ser elevado por el corte superior descubierto de estas plumas sub-apicales y la presencia de las cámaras húmedas que impidió una adecuada ventilación. Por otro lado, el alto porcentaje de prendimiento obtenido con las plumas apicales confirman el éxito de este tipo de estructuras en los micro y mini-injertos de maracuyá amarillo (Santos et al., 2014; Oliari et al., 2016), y que es bastante acertada la preferencia de los agricultores de usar las partes apicales y con más de tres yemas axilares de brotes de maracuyá para realizar los injertos (Araujo et al., 2004).

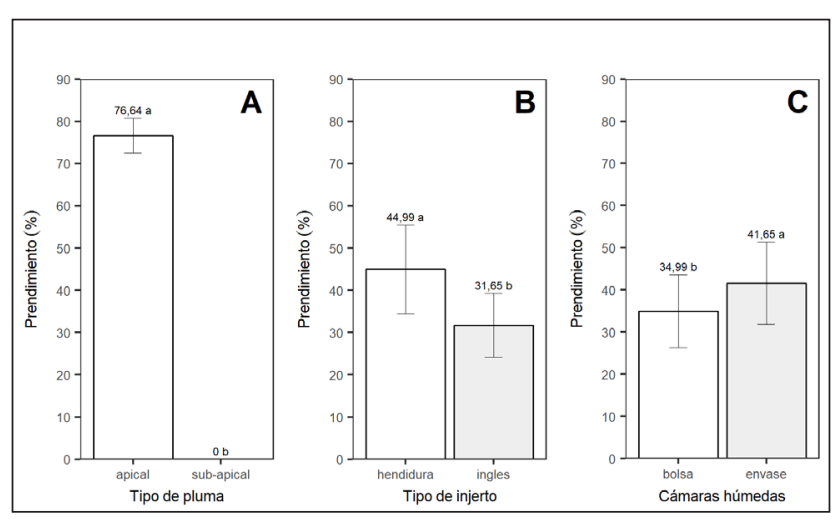

Figura 2. Efectos simples de los factores en el porcentaje de prendimiento de los injertos de granadilla sobre maracuyá, 32 días después de realizados. A: Tipo de pluma, B: Tipo de injerto, C: Cámara húmeda

En lo que respecta al tipo de injerto, se observa que el injerto de hendidura (44,99\%) fue mejor que el inglés simple $(35,65 \%)$. De acuerdo a lo expresado por Ruggiero (1991), los dos tipos de injerto son los más empleados para maracuyá amarillo y con ambos se logra más de $90 \%$ de prendimiento. Igualmente Silva et al., 2005 estudiando el empleo de injertos de mesa de maracuyá amarillo sobre Passiflora alata usando estacas para los portainjertos y plumas para los injertos, ambos a partir de plantas de año y medio de edad, no encontraron diferencias entre los dos tipos de injerto; más tarde con las mismas especies pero empleando el método hipocotiledonar, Santos et al. (2011) arriban a las mismas conclusiones, alcanzando ambos tipos de injerto, $98 \%$ de prendimiento. En otros trabajos experimentales en los cuales solo se trabajó con injerto de hendidura se reportan elevados porcentajes de éxito; así, Alexandre (2013) con mini-injertos empleando como portainjerto al maracuyá amarillo y como copa varias especies de Passiflora, obtuvo entre 68 y $90 \%$ de prendimiento, Nogueira et al. (2011) injertando con el método hipocotiledonar, maracuyá amarillo sobre siete especies de Passiflora reporta hasta $100 \%$ de éxito. Otros 
varios trabajos informan de resultados similares altamente positivos de este tipo de injerto (Lima, 2005; Chaves et al., 2004; Silva et al., 2005). Por otro lado, trabajando solo con injerto inglés simple, Santos et al., (2014) empleando a Passiflora cincinnata y $P$, alata como portainjertos de maracuyá amarillo, rojo y dulce encontraron entre 41,6 y $51,8 \%$ de prendimiento con el portainjerto $P$. cincinnata y entre 75 y $91 \%$ con $P$. alata. Posiblemente las diferencias encontradas entre los dos tipos de injerto en la presente investigación, sean consecuencia solamente de la mayor dificultad para poner en contacto los tejidos de los cortes a bisel efectuados y su posterior atadura que supone el injerto inglés, en comparación con el injerto de hendidura.

En la evaluación del tipo de cámara húmeda, los envases rígidos de bebidas dieron mejores resultados que las bolsas de polietileno (41,65 y 34,99 \%, respectivamente). Los reportes referidos a los mini injertos y a los injertos hipocotiledonares realizados mayormente con maracuyá amarillo, indican que el uso de cámaras húmedas, constituidas en todos los casos por bolsas de plástico transparente, es indispensable para crear un ambiente de alta humedad que impida la deshidratación de los segmentos apicales utilizados como plumas y que los mantengan vivos mientras ocurre la soldadura con el portainjerto (Correa et al., 2010; Silva et al., 2015; Nogueira et al., 2011; Oliari et al., 2016; Alexandre et al., 2013). Los envases de bebida tuvieron un mayor diámetro y altura que las bolsas plásticas que permitió acomodar mejor las estructuras injertadas e igualmente es muy posible que haya habido una mejor conservación de la alta humedad en su interior ya que no tuvo la base libre y ventilada como fue el caso de las bolsas plásticas.

Longitud de brote del injerto

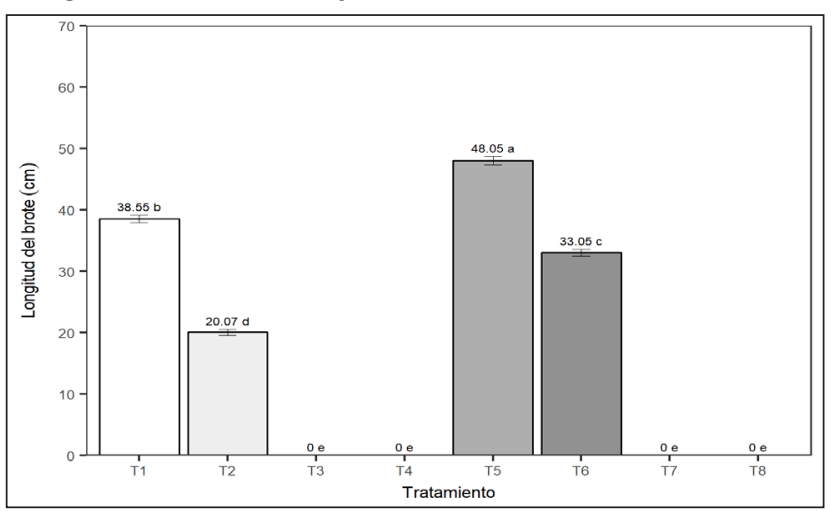

Figura 3. Longitud promedio (cm) del brote de los injertos de granadilla sobre maracuyá. 67 días después de efectuados. Interacción de forma de injerto, tipo de pluma y tipo de cámara húmeda

T1: injerto hendidura + pluma apical + bolsa de polietileno, T2: injerto inglés simple + pluma apical + bolsa de polietileno, T3: injerto hendidura + pluma sub-apical + bolsa de polietileno, T4: injerto inglés simple + pluma sub-apical + bolsa de polietileno, T5: injerto hendidura + pluma apical + envase de bebida, T6: injerto inglés simple + pluma apical + envase de bebida, T7: injerto Hendidura + pluma sub-apical + envase de bebida, T8: injerto inglés simple + pluma sub-apical + envase de bebida.
Como consecuencia de la falta total de éxito en los injertos realizados con las plumas sub-apicales, en los tratamientos T3, T4, T7 y T8 no hubo registros de longitud de los brotes, como se observa en la Figura 3. En el resto de tratamientos la tendencia de este parámetro fue similar al de los porcentajes de prendimiento, destacando el tratamiento T5 con 48,05 cm, seguido por T1, T6 y T2 con $38,55,33,05$ y $20,07 \mathrm{~cm}$, solo que esta vez todas las diferencias entre ellos tuvieron significación estadística.

En cuanto a los efectos simples o promedios de los factores, en la Figura 4 se aprecia que las plumas apicales dieron como resultado brotes de $34,93 \mathrm{~cm}$. El injerto de hendidura con $21,65 \mathrm{~cm}$ fueron mejores que el inglés simple que alcanzó un promedio de13,28 cm. Posiblemente con los injertos de hendidura la soldadura de las partes ocurrió antes por lo que el nuevo crecimiento tuvo la ventaja de empezar a desarrollar antes que el brote de los injertos inglés simple. Con la cámara húmeda confeccionada con envases de bebidas, el promedio de brotes de $20,27 \mathrm{~cm}$ fue superior a la bolsa de polietileno que registró 14,65 $\mathrm{cm}$. Es posible que el tamaño más reducido de las bolsas plásticas haya sido un impedimento físico que perturbó el incremento en longitud del brote generado. Al respecto, Silva et al (2015) estudiando tres formas de injerto y el empleo de bolsas plásticas como cámaras húmedas en maracuyá amarillo encontraron que hubo un efecto positivo de las cámaras húmedas en el prendimiento de los injertos, pero a su vez produjeron una reducción en el crecimiento; otras investigaciones en otras especies concluyen que las bolsas plásticas a usar como cámaras húmedas deben de ser de mayor tamaño para que no interfieran en el desarrollo de los brotes o en todo caso que se utilicen por un menor tiempo (Jacomino et al., 2000).

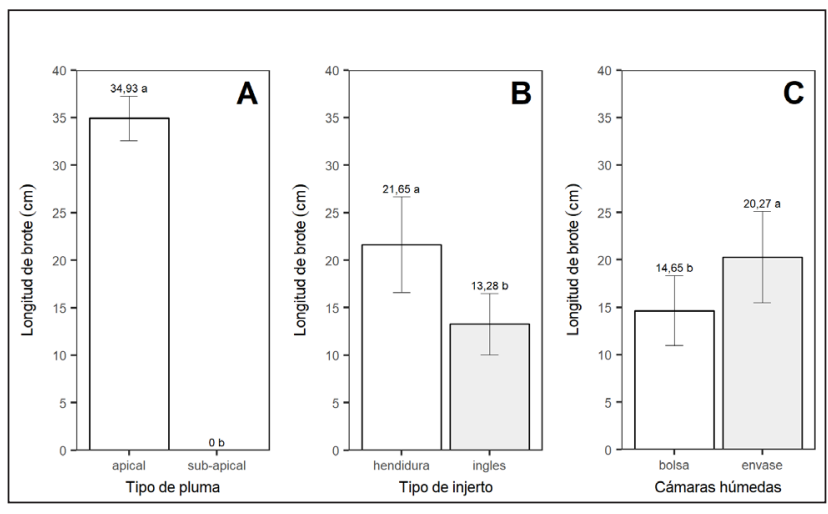

Figura 4. Efectos simples de los factores en la longitud del brote $(\mathrm{cm})$ de los injertos de granadilla sobre maracuyá, 67 días después de realizados. A: Tipo de pluma, B: Tipo de injerto, C: Cámara húmeda

\section{Conclusiones}

El prendimiento de los injertos con plumas sub-apicales fue nulo. Las únicas que dieron resultados positivos fueron las apicales. El injerto de hendidura funcionó mejor que el inglés simple en el prendimiento de los injertos y el 
crecimiento del brote. La cámara húmeda individual confeccionada con envases plásticos de bebidas fue mejor que las bolsas plásticas para los dos parámetros evaluados.

\section{Literatura citada}

Alexandre, R.; Lopes, J.; Tiradentes, A.; Bruckner C. and Otoni, W. 2013. Metodología de minienxertia em maracujazeiros. Rev. Bras. Frutic. 35(1): 329-332.

Araújo, F. de; Santos, C. and Lelo, F. 2004. Propagação vegetativa do maracujá-do-mato: espécie resistente à seca, de potencial econômico para agricultura de sequeiro. Petrolina: Embrapa Semiárido, 2004. (Instruções Técnicas).

Cárdenas, J. 2011. Morfología y tratamientos pregerminativos de semillas de granadilla (Passiflora ligularis Juss). Tesis Magister en Ciencias Agrarias. Universidad Nacional de Colombia. Bogotá. 92p.

Cerdas, M. y Castro, J. 2003. Manual práctico para la producción, cosecha y manejo pos cosecha del cultivo de granadilla (Passiflora ligularis, Juss). Ministerio de Agricultura y Ganaderia, San José (Costa Rica). 63 p.

Chaves, R. da C.; Junqueira, N. T. V.; Manica, I.; Peixoto, J. R.; Pereira, A. V. and Fialho, J. de F. 2004. Enxertia de maracujazeiro-azedo em estacas herbáceas enraizadas de espécies de passifloras nativas. Revista Brasileira de Fruticultura, Jaboticabal - SP, 26(1): 120-123.

Corrêa, L. de S.; Cavichioli, J. C.; Oliveira, J. C. de. and Boliani, A. C. 2010. Uso de câmara úmida em enxertia convencional de maracujazeiro-amarelo sobre três portaenxertos. Revista Brasileira de Fruticultura, Jaboticabal - SP, 32(2):591-598.

Esquerre-Ibañez B.; C. Rojas-Idrogo, S. Llatas-Quiroz \& G. Delgado-Paredes. 2014. El género Passiflora L. (Passifloraceae) en el Departamento de Lambayeque, Perú. Act. Bot. Malac. 39: 55-70.

Jacomino, AP; Minami, K; Scarpare Filho, JA. and Kluge, RA. 2000. Grafting processes for mango tree protection. Scientia Agricola, 57(1):105-108.

Lima, A. de A. 2005. Aspectos fitotécnicos: desafíos da pesquisa. In: Faleiro, F.G.; Junqueira, N.T.V.; Braga, M. F. (Ed.). Maracujá: germoplasma e melhoramento genético. Planaltina Distrito Federal: Embrapa Cerrados. 670 p.

Maicelo, J.; Guevara, E.; Barboza, E. y Oliva, S. 2017. Evaluación de tres tipos de injertos de granadilla sobre maracuyá con púas producidas en medio hidropónico y en sustrato sólido, Chachapoyas. Rev. de investig. agroproducción sustentable, 1(1):70-79.

Nogueira, G.; Roncatto, G.; Ruggiero, C.; Oliveira, J. e Malheiros, E. 2011. Produção de mudas de maracujazeiro-amarelo por enxertia hipocotiledonar sobre sete espécies de Passifloras. Revista Brasileira de Fruticultura, Jaboticabal, 33(1): 237-245.

Oliari, L. S.; Giles, J. A. D.: Maynrick, L. G.; Oliveira, J. P. B.; Lopes, J. C.; Otoni, W. C.; Schmildt, E. R.;
Aoyama, E. M. and Alexandre, R. S. 2016. Mini-grafting of adult Passiflora edulis Sims f. flavicarpa Deg. scions onto vegetatively propagated adult rootstocks of P. mucronata Lam. Australian Journal of Crop Science, 10 (4): 490-496.

Ruggiero, C. y Oliveira, J.C. de. 1998. Enxertia do maracujazeiro. In: Simpósio Brasileiro Sobre a Cultura do Maracujazeiro, 5., 1998. Anais... Jaboticabal: FUNEP, 70-92p.

Ruggiero, C. 1991. Enxertia do maracujazeiro. In: São José, A. R. (Ed.). A cultura do maracujá no Brasil. Jaboticabal: FUNEP, 43-59p.

Santos, V.; Ramos, J.; Oliveira, M. y Silva, E. 2011. Tipos de enxertia em diferentes idades de plantas de maracujazeiro. Rev. Bras. Frutic., Jaboticabal, 33(4):1359-1363.

Santos, V.; Ramos, J.; Chagas, E.; Dias, M.; Locatelli, G. e De Oliveira, M. 2014. Enxertia de diferentes combinações de copas e porta-enxertos em maracujazeiros. Semina: Ciências Agrárias, Londrina, 35(3):1201-1208.

Silva, R.; De Aguiar, A.; Mendonça, V.; Cardoso, E. e García, K. 2015. Produção de mudas de maracujazeiro amarelo com diferentes tipos de enxertia e uso da câmara úmida. Revista Verde (Pombal - PB - Brasil), 10(4):64 - 68.

Silva, F.M.; Correa, L. de S.; Boliani, A.C. and Santos, P. C. dos. 2005. Enxertia de mesa de Passiflora edulis Sims f. flavicarpa Deg. sobre Passiflora alata Curtis, em ambiente de nebulização intermitente. Revista Brasileira de Fruticultura, Jaboticabal - SP, 27(1): 98101.

Zucareli V.; Ono, E.; Boaro, C.; Brambilla, W. 2014. Desenvolvimento inicial de maracujazeiros (Passiflora edulis f. flavicarpa, Passiflora edulis e Passiflora alata) enxertados sobre Passiflora cincinnata. Semin. Ciênc. Agr. 35(5): 2325-2340. 